\title{
Opacity of the Microwave Background Radiation to Ultrahigh Energy Cosmic Rays
}

\author{
L.A. Anchordoquia ${ }^{a}$ M.T. Dova $^{a *}$, L.N. Epele ${ }^{a}$, and J.D. Swain ${ }^{b}$ \\ ${ }^{a}$ Departamento de Física, Universidad Nacional de La Plata C.C. 67, (1900) La Plata, Argentina \\ ${ }^{b}$ Department of Physics, Northeastern University, Boston, Massachusetts, 02115, USA
}

In this work we re-examine the influence of the microwave background radiation on the propagation of extremely high energy cosmic rays. We present a recalculation of the rate of fractional energy loss in the continuous energy loss approximation including the effects of resonances other than the $\Delta$. We also discuss the distortion of the cosmic ray spectrum due to interactions with the relic photons.

As soon as the microwave background radiation (MBR) was discovered [1], it became unavoidable to evaluate the interaction of ultrahigh energy cosmic rays (UHECR's) with the relic photons. This interaction gives rise to a cutoff in the cosmic ray spectrum at energies $>5 \times 10^{19} \mathrm{eV}$, as first shown in 1966 by Greisen, Zatsepin and Kuz'min, and then called the "GZK cutoff" $[2,3]$.

Recent observations with several air shower arrays have clearly detected a few UHECR's with energies above $10^{20} \mathrm{eV}[4-7]$. Since the GZK cutoff implies relative proximity of such UHECR sources, the origin of such energetic particles has became one of the most pressing questions of cosmic ray astrophysics.

The study of the modification of the spectrum due to interactions with the MBR en route to Earth, is essential if one is to obtain a deeper understanding of the origin of the highest energy cosmic rays. There exist in the literature various works on the propagation of nucleons in the intergalactic medium. [8-17]. In the present work we re-analyze the effects of meson photoproduction, the dominant energy loss mechanism, using the continuous energy loss approximation which is expected to be reasonable for the problem at hand. This allows us to discuss, in a general way, the modification of the spectrum of cosmic rays from nearby sources.

The propagation of cosmic rays through the

\footnotetext{
*E-mail: dova@venus.fisica.unlp.edu.ar
}

intergalactic medium is described by a kinetic transport equation which takes into account three mechanisms for energy losses: redshift, pair production, and pion photoproduction. For nearby sources corresponding to propagation times $\tau \approx$ $3 \times 10^{8}$ years, redshift effects can be neglected.

If one assumes that the highest energy cosmic rays are indeed nucleons, the fractional energy loss due to interactions with the cosmic background radiation at temperature $T$ and redshift $z=0$, is determined, following the formalism of [18], by

$$
\begin{aligned}
&-\frac{1}{E} \frac{d E}{d t}=-\frac{c k T}{2 \pi^{2} \Gamma^{2}(c \hbar)^{3}} \times \\
& \int_{w_{0}}^{\infty} d w_{r} \sigma\left(w_{r}\right) K\left(w_{r}\right) w_{r} \ln \left(1-e^{-w_{r} / 2 \Gamma k T}\right)
\end{aligned}
$$

where $w_{r}$ is the photon energy in the rest frame of the nucleon, $w_{0}$ is the threshold energy for the reaction, $K\left(w_{r}\right)$ is the average fraction of the energy lost by the photon to the nucleon in the rest frame, $\sigma\left(w_{r}\right)$ is the total cross section of interaction and $\Gamma$, as usual, is the nucleon Lorentz factor.

The characteristic time for the energy loss due to pair production at $E>10^{19} \mathrm{eV}$ is $t \approx 5 \times 10^{9} \mathrm{yr}$ [19] and therefore it does not affect the spectrum of nucleons arriving from nearby sources. Consequently, for nucleons with $E \geq 3 \times 10^{19} \mathrm{eV}$, meson photoproduction is the dominant mechanism for energy loss.

In order to determine the effect of meson pho- 
toproduction on the spectrum of cosmic rays it is necessary to study the kinematics of photonnucleon interactions. Assuming that reactions mediated by baryon resonances have spherically symmetric decay angular distributions $[13,16]$, the average energy loss of the nucleon after $n$ resonant collisions is given by

$\langle K\rangle=1-\frac{1}{2^{n}} \prod_{i=1}^{n}\left(1+\frac{m_{R_{i}}^{2}-m_{M}^{2}}{m_{R_{i-1}}^{2}}\right)$

where $m_{R_{i}}$ denotes the mass of the resonant system of the chain, $m_{M}$ the mass of the associated meson, $m_{R_{0}}=\sqrt{s}$ is the total energy of the reaction in the centre of mass and $m_{R_{n}}$ the mass of the nucleon. It is well established from experiments that at very high energies $(\sqrt{s}$ above $\sim 3 \mathrm{GeV}$ ), the incident nucleons lose half their energy via pion photoproduction independent of the number of producted pions.

In the region dominated by baryon resonances, the cross section is obtained as a sum of BreitWigner distributions constructed from the experimental data in the Table of Particle Properties [20] considering the main resonances produced in $N \gamma$ collisions with $\pi N, \pi \pi N$, and $\pi K N$ final states. For the cross section at high energies we used the fits to the high-energy cross section $\sigma_{\text {total }}(p \gamma)$ made by the CERN-HERA and COMPAS Groups [20]. In this energy range, the $\sigma_{\text {total }}(n \gamma)$ is to a good approximation identical to $\sigma_{\text {total }}(p \gamma)$.

We perform the numerical integration of (1), taking into account the aforementioned resonance decays and the production of multipion final states at higher centre of mass energies $(\sqrt{s} \sim 3$ $\mathrm{GeV})$. The $\chi^{2}$ fit of the numerical result of (1) to an exponential $A \exp (-B / E)$, similar to that proposed by Berezinsky and Grigor'eva [10] for the region of resonances, gives $A=(3.66 \pm 0.08) \times$ $10^{-8} \mathrm{yr}^{-1}, B=(2.87 \pm 0.03) \times 10^{20} \mathrm{eV}$. At high energies the fractional energy loss was fitted with a constant, $C=(2.42 \pm 0.03) \times 10^{-8} \mathrm{yr}^{-1}$. These results are different from those obtained in [10], due to a refined expression for the total cross section. From the values determined for the fractional energy loss, it is straightforward to compute the energy degradation of UHECR's in terms of their flight distance. The results shown in Fig 1 , are consistent with those previously obtained by Cronin [21], in which only single pion production is considered. Our results therefore support the assumption, that the energy loss is dominated by single pion photoproduction.

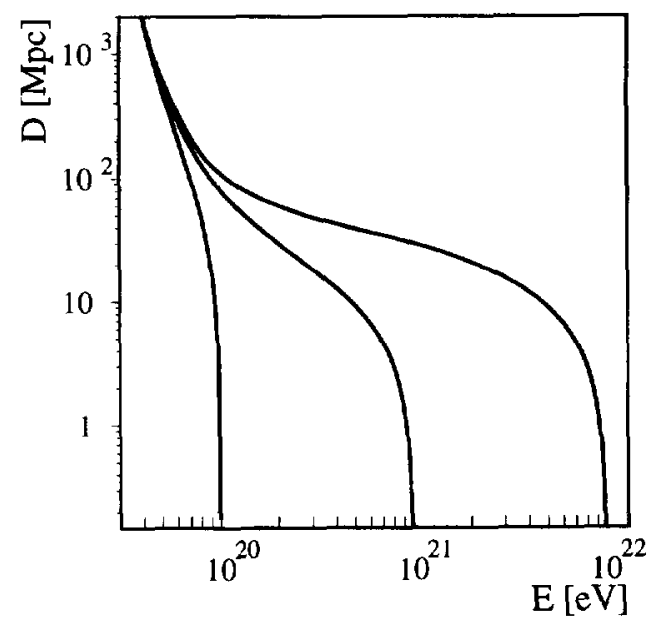

Figure 1. Energy attenuation length of nucleons in the intergalactic medium for initial energies of $10^{20}, 10^{21}$ and $10^{22} \mathrm{eV}$

Let us now turn to the modification that the MBR produces in UHECR spectrum. We assume that nucleons propagate in a straight line through the intergalactic medium, the evolution of the spectrum $N(E, t)$ is governed by the following balance equation

$\frac{\partial N}{\partial t}=\frac{\partial(b(E) N)}{\partial E}+D \nabla^{2} N+Q$

that takes into account the conservation of the total number of nucleons in the spectrum. In the first term on the right, $b(E)$ is the mean rate at which particles lose energy. The second term, the diffusion in the MBR, may be neglected due to the low density of the relic photons. The third term corresponds to the particle injection rate into the intergalactic medium (the sources).

Let us focus our attention on the evolution of a single source spectrum. In this case, the solution 
of eq. (3) reads [11],

$N(E, t)=\frac{1}{b(E)} \int_{E}^{\infty} Q\left(E_{g}, t^{\prime}\right) d E_{g}$

with

$t^{\prime}=t-\int_{E}^{E_{g}} \frac{d \tilde{E}}{b(\tilde{E})}$

and $E_{g} \equiv$ energy of the nucleon when emitted by the source. As there exists evidence that the source spectrum of cosmic rays has a power-law dependence $Q(E)=\kappa E^{-\gamma}$ (see for example [14]), the injection spectrum of a single source can be written as $Q(E, t)=\kappa E^{-\gamma} \delta\left(t-t_{0}\right)$. At very high energies, i.e. where $b(E)=C E$, the total number of particles at a given distance from the source is given by

$$
\begin{aligned}
N(E, t) \approx & \frac{\kappa}{b(E)} \times \\
& \int_{E}^{\infty} E_{g}^{-\gamma} \delta\left(t-\frac{1}{C} \ln \frac{E_{g}}{E}\right) d E_{g}
\end{aligned}
$$

or equivalently

$N(E, t) \approx \kappa E^{-\gamma} e^{-(\gamma-1) C t}$

This means that the spectrum is uniformly damped by a factor depending on the proximity of the source.

At low energies, in the region dominated by baryon resonances, the parametrisation of $b(E)$ does not allows a complete analytical solution. However using the change of variables

$\tilde{t}=\int_{E}^{E_{g}} \frac{d \tilde{E}}{b(\tilde{E})}$

with $E_{g}=\xi(E, \tilde{t})$ and $d \tilde{t}=d E_{g} / b\left(E_{g}\right)$, we easily obtain,

$$
\begin{aligned}
N(E, t)= & \frac{\kappa}{b(E)} \times \\
& \int_{0}^{\infty} \xi(E, \tilde{t})^{-\gamma} \delta(\tilde{t}-t) b[\xi(E, \tilde{t})] d \tilde{t}
\end{aligned}
$$

and then, the compact form,

$$
N(E, t)=\frac{\kappa}{b(E)} E_{g}^{-\gamma} b\left(E_{g}\right)
$$

In our case, where we have assumed an exponential behaviour of the fractional loss energy,
$E_{g}$ is fixed by the constraint: $A t-\operatorname{Ei}(B / E)+$ $\mathrm{Ei}\left(B / E_{g}\right)=0, \mathrm{Ei}$ being the exponential integral $[22]$.

Studies are underway of the case of a nearby source for which $E_{g} \approx E+\Delta E$. In this case it is possible to obtain an analytical solution. An expansion of $b(E+\Delta E)$ in powers of $\Delta E$ in eq. (5) allows one to obtain an expression for $\Delta E$ :

$\Delta E \approx\left\{\exp \left[\frac{t b(E)(E+B)}{E^{2}}\right]-1\right\} \frac{E^{2}}{(E+B)}$

To describe the modification of the spectrum, it is convenient to introduce the factor $\eta$, the ratio of the modified spectrum to the unmodified one. At low energy, the value is

$\eta=\left(\frac{E+\Delta E}{E}\right)^{-\gamma} \frac{b(E+\Delta E)}{b(E)}$

Equations (11) and (12) describe the spectral modification factor up to energies of $\approx 95 \mathrm{EeV}$ $(\approx 85 \mathrm{EeV})$ with a precision of $6 \%(20 \%)$ for a source situated at $50 \mathrm{Mpc}(100 \mathrm{Mpc})$.

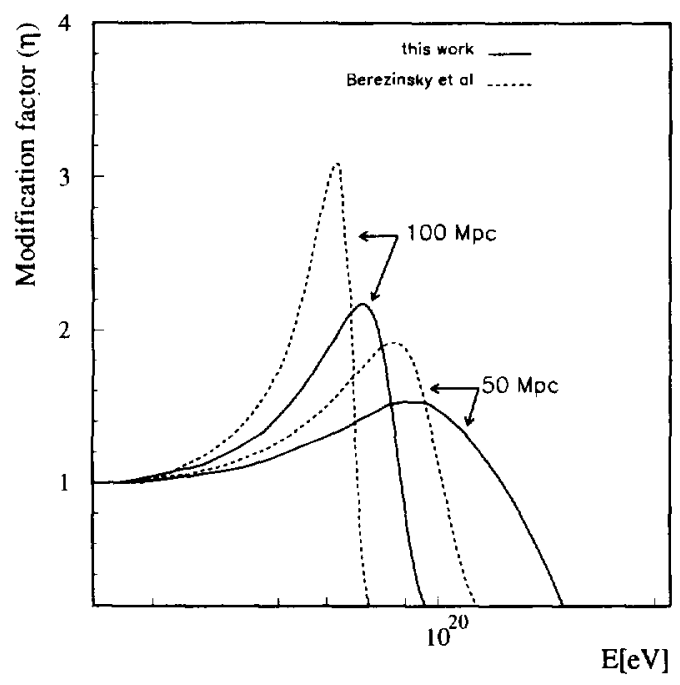

Figure 2. Modification factor of single-source energy espectra for different values of propagation distance and power law index $\gamma=2.5$.

In Fig. 2 we plot the modification factors for the case of nearby sources with power law injection $(\gamma=2.5)$ to compare with the corresponding results of ref.[10]. The most significant features 
of $\eta$ are the bump and the cutoff. It has been noted in the literature that the continuous energy loss approximation tends to overestimate the size of the bump [12,17]. Fig. 2 shows the bump is less pronounced in our treatment. As the bump is a consequence of the sharp dependence of the nucleon free path on energy, we attribute these differences to the replacement of a cross section approximated by the values at threshold energy [10], by a more detailed expression taking into account the main baryon resonances.

These results allow us to recover the continuous energy loss approximation which is the only way in which analytical solutions can be obtained for a wide range of situations.

\section{Acknowledgments}

We are grateful to Huner Fanchiotti and Carlos García Canal for illuminating discussions, and to James Cronin for stimulating our initial interest in this subject. One of us (MTD) would also like to thank the organizing committee for a most enjoyable and interesting conference.

\section{REFERENCES}

1. A. A. Penzias and R. W. Wilson, $A p . J 142$, 419 (1965).

2. K. Greisen, Phys. Rev. Lett. 16, 748 (1966).

3. G. T. Zatsepin and V. A. Kuz'min, Pis'ma Zh. Eksp. Teor. Fiz. 4, 114 (1966).

4. S. Yoshida et al., Astropart. Phys. 3, 105 (1995).

5. N. Hashashida et al., Phys. Rev. Lett. 73, 3491 (1994).

6. D. J. Bird et al., Phys. Rev. Lett. 71, 3401 (1993).

7. D. J. Bird et al., Ap. J. 441, 144 (1995).

8. V. S. Berenzinsky and G. T. Zatsepin, Yad. Fiz. 13, 797 (1971).

9. F. W. Stecker, Phys. Rev. Lett. 21, 1016 (1968).

10. V. S. Berezinsky and S. I. Grigor'eva, Astron. Astrophys. 199, 1 (1988).

11. F. W. Stecker, Nature 342, 401 (1989).

12. F. A. Aharonian, B. L. Kanevsky and V. V. Vardanian, Astrophys. Space Sci. 167, 93
(1990).

13. J. P. Rachen and P. L. Biermann, Astron. Astrophys. 272, 161 (1993).

14. C. T. Hill and D. N. Schramm, Phys. Rev. D 31, 564 (1985).

15. S. Yoshida and M. Teshima, Prog. Theor. Phys. 89, 833 (1993).

16. F. A. Aharonian and J. W. Cronin, Phys. Rev. D 50, 1892 (1994).

17. S. Lee, FERMILAB-Pub-96/066-A.

18. V. S. Berenzinsky, Yad. Fiz. 11, 399 (1970).

19. G. Blumenthal, Phys. Rev. D 1, 1596 (1970).

20. Review of Particles Properties, Phys. Rev. D 50, 1335 (1994).

21. J. W. Cronin, Nucl. Phys. B (Proc. Suppl.) 28B, 213 (1993). See also reference [16].

22. M. Abramowitz and I. A. Stegun, "Handbook of Mathematical Functions" (New York, Dover, 1970). 